hep-th/0204031

UT-02-17

\title{
Gauge transformations on a D-brane in Vacuum String Field Theory
}

\author{
Yosuke Imamura円 \\ Department of Physics, Faculty of Science, University of Tokyo \\ Hongo 7-3-1, Bunkyo-ku, Tokyo 113-0033, Japan
}

\begin{abstract}
We study gauge transformations of the Hata-Kawano vector state on a D25-brane in the framework of vacuum string field theory. We show that among the infinite number of components of the polarization vector, all the components except one spacetime vector degree of freedom are gauge freedom, and give string field gauge transformations reproducing gauge transformations of the constant modes of the $U(1)$ gauge field. These gauge transformations can be used to fix the normalization of the vector field. We also discuss a difficulty in obtaining a gauge invariant action of the vector field. Our arguments rely on the factorization ansatz of gauge transformations.
\end{abstract}

\footnotetext{
${ }^{1}$ E-mail: imamura@hep-th.phys.s.u-tokyo.ac.jp
} 


\section{Introduction}

Two kinds of cubic open string field theories, Witten's cubic string field theory [1] and vacuum string field theory [2, 3, 田, 5], have been recently investigated. These are considered as two descriptions of one theory covering both perturbative and nonperturbative vacua. Although it is expected that these two theories are related to each other by some (singular) string field redefinition, derivation of one theory from the other has not been succeeded. Now we are at a stage of collecting evidences of the equivalence of these theories.

In Witten's string field theory, which describes open strings on D25-branes, the nonperturbative vacuum state was constructed by the level truncation method, and the difference of the energy densities between perturbative and nonperturbative vacua was computed numerically with high precision agreement with the expected D25-brane tension[6, 7]. This result was confirmed analytically in the framework of boundary string field theory [8]. Numerical studies of the BRS cohomology at the nonperturbative vacuum suggested the decoupling of the physical modes [9, 10].

On the other hand, in vacuum string field theory, D-branes are represented as non-trivial solutions of the equation of motion. The action of vacuum string field theory is

$$
S[\Phi]=-\frac{1}{g_{\mathrm{v}}}\left(\frac{1}{2} \Phi \cdot Q \Phi+\frac{1}{3} \Phi \cdot(\Phi * \Phi)\right),
$$

where $Q$ is a pure ghost operator and $g_{\mathrm{v}}$ is a coupling constant. The explicit forms of solutions representing $\mathrm{D} p$-branes $\Xi_{p}$ with arbitrary dimensions $p$ are known[11, 3]. By substituting these solutions to the action (11) we can obtain their energy densities $\mathcal{E}_{p}$. Numerical[3] and analytical[12] computations of the ratio $\mathcal{E}_{p} / \mathcal{E}_{p+1}$ showed that it coincides with the expected result $T_{p} / T_{p+1}=2 \pi$.

An attempt to determine the overall factor of the D25-brane energy density was first done by Hata and Kawano 13. They first constructed a string field solution representing the tachyon field on a D-brane as a fluctuation mode of the sliver state $\Xi$, and computed the three tachyon vertex to fix the ratio between $g_{\mathrm{v}}$ in the action (1) and the perturbative coupling constant $g_{\mathrm{str}}$. Disappointingly, the ratio $\mathcal{E}_{25} / T_{25}=(2 \pi)^{25} g_{\text {str }} \mathcal{E}_{25}$ did not coincide with the expected result 1 . Although the numerical computation was improved in [14, the result is close to 2 rather than 1 . This unwelcome result was later confirmed analitically [15].2]

Hata and Kawano also constructed the massless vector field on a D25-brane. In this paper, we study gauge transformations of this Hata-Kawano (HK) vector state. The HK vector state includes a polarization vector $d_{m}^{\mu}$ with a Lorentz index $\mu$ and a level index $m$. Due to the level index $m$, this has too many components to represent a vector field. We will show that, for each spacetime direction, only

\footnotetext{
${ }^{2}$ After this work was finished a paper [23] appeared where some progress in this popic is made.
} 
one of infinite number of components corresponds to the $U(1)$ vector field on a Dbrane and all the others are gauge degrees of freedom. Furthermore, we present a string field gauge transformation reproducing a gauge transformation of the constant modes of the gauge field (Wilson lines). This gauge transformation can be used to fix the normalization of the gauge field.

Our original motivation was to extract the coupling constant $g_{\text {str }}$ from the kinetic term of the normalized vector field and to check the coincidence between the energy density of the sliver state and the expected D25-brane tension. However, due to a certain problem, we could not obtain any information of the coupling constant $g_{\text {str }}$. We will discuss this problem in the last section.

We should notice that our arguments in this paper completely rely on the factorization ansatz of gauge transformations as will be mentioned in the next section. At this point, however, we have not shown the existence of such gauge transformations. If gauge transformations could not be factorized, our arguments given below might be spoiled.

Before ending this section, we define several matrices and vectors used in this paper. In the oscillator formalism, the 3-string vertex is given by 16, 17]

$$
\begin{aligned}
& \left|V_{3}\right\rangle_{\mathrm{mat}}=e^{E}\left|p_{1}\right\rangle\left|p_{2}\right\rangle\left|p_{3}\right\rangle \\
& E=\sum_{r, s=0}^{3} \sum_{m, n=1}^{\infty}\left(-\frac{1}{2} \eta_{\mu \nu} a_{m}^{\dagger r \mu} V_{m n}^{r s} a_{n}^{\dagger s \nu}-\eta_{\mu \nu} p^{r \mu} V_{0 n}^{r s} a_{n}^{\dagger s \nu}-\frac{1}{2} \eta_{\mu \nu} V_{00} p^{r \mu} p^{r \nu}\right) .
\end{aligned}
$$

The coefficient matrices $V^{r s}$ is called Neumann coefficients. We define the following matrices and vectors.

$$
\begin{gathered}
M_{m n}=\left(C V^{r r}\right)_{m n}, \quad\left(M_{+}\right)_{m n}=\left(C V^{r r+1}\right)_{m n}, \quad\left(M_{-}\right)_{m n}=\left(C V^{r r-1}\right)_{m n}, \\
v_{n}=V_{0 n}^{r r}, \quad\left(v_{+}\right)_{n}=V_{0 n}^{r r+1}, \quad\left(v_{-}\right)_{n}=V_{0 n}^{r-1}, \quad(n \geq 1) . \\
v_{1}=v_{+}-v_{-}, \quad v_{ \pm 0}=v_{ \pm}-v .
\end{gathered}
$$

where $C_{m n}=\delta_{m n}(-1)^{n}$ is the twist matrix. The Lorentz metric $\eta_{\mu \nu}$ and level indices $m, n, \ldots$ are often suppressed.

\section{Factorization ansatz of gauge transformations}

In vacuum string field theory, D-branes are represented as classical solutions of an equation of motion. The equation of motion obtained from the action (11) is

$$
Q \Phi+\Phi * \Phi=0
$$

Because operator $Q$ is pure ghost there exist solutions factored into matter part $\Phi^{\text {mat }}$ and ghost part $\Phi^{\text {gh }}$ :

$$
\Phi=\Phi^{\mathrm{mat}} \otimes \Phi^{\mathrm{gh}}
$$


In this paper, we always assume this factorization. $\Phi^{\text {mat }}$ and $\Phi^{\mathrm{gh}}$ satisfy the following equations.

$$
\begin{aligned}
& Q \Phi^{\mathrm{gh}}+\Phi^{\mathrm{gh}} * \Phi^{\mathrm{gh}}=0, \\
& \Phi^{\mathrm{mat}}=\Phi^{\mathrm{mat}} * \Phi^{\mathrm{mat}}
\end{aligned}
$$

Although there are several known solutions for the ghost part $\Phi^{\mathrm{gh}}$ 18], we will not specify $\Phi^{\mathrm{gh}}$ and concentrate on the matter part.

The action (1) is invariant under the following gauge transformation.

$$
\delta \Phi=Q \Lambda+\Phi * \Lambda-\Lambda * \Phi .
$$

For our purpose, 'finite' gauge transformations are also convenient.

$$
\Phi=U^{-1} * Q U+U^{-1} * \Phi^{\prime} * U .
$$

We ignore the topological term $\delta S \sim\left(U^{-1} * Q U\right)^{3}$ because we consider only gauge transformations generated by infinitesimal ones. The proof of the gauge invariance under (12) is analogous to that of three dimensional Chern-Simons theories. However, in string field theory, we need additional assumption, which is trivial in the Chern-Simons theories. We have to assume the existence of a string field $\mathcal{I}$ satisfying

$$
Q \mathcal{I}=0, \quad \mathcal{I} * \Phi=\Phi * \mathcal{I}=\Phi,
$$

for an arbitrary $\Phi$, and $U^{-1}$ have to be defined from $U$ by $U * U^{-1}=\mathcal{I}$. We are now assuming the factorization (8). If we require the gauge transformation not to break this factorization, we need to assume that $\mathcal{I}$ also factorizes into matter part $\mathcal{I}^{\text {mat }}$ and ghost part $\mathcal{I}^{\text {gh }} \llbracket$. We can identify $\mathcal{I}^{\text {mat }}$ as the matter identity $I^{\text {mat }}$. The ghost part $\mathcal{I}^{\text {gh }}$ has to satisfy

$$
Q \mathcal{I}^{\mathrm{gh}}=0, \quad \mathcal{I}^{\mathrm{gh}} * \Phi^{\mathrm{gh}}=\Phi^{\mathrm{gh}} * \mathcal{I}^{\mathrm{gh}}=\Phi^{\mathrm{gh}},
$$

where $\Phi^{\text {gh }}$ is the ghost part of $\Phi$ in (13). Naively, the ghost identity $I^{\text {gh }}$ satisfies the second equation in (14). It is known that $Q I^{\text {gh }}=0$ if we use $\zeta$ function regularization. Even with other regularization, $Q I^{\text {gh }}$ is null state, which has vanishing product with any string field 18, 19]. However, precisely speaking, there is no identity field satisfying $I^{\mathrm{gh}} * \Phi^{\mathrm{gh}}=\Phi^{\mathrm{gh}} * I^{\mathrm{gh}}=\Phi^{\mathrm{gh}}$ for any string field $\Phi^{\text {gh }}$ [18, 19]. Even the ghost identity defined in [17] does not satisfy this condition. Because of the universality of the ghost factor this identity condition may unnecessarily be tight. For our argument below $\mathcal{I}^{\text {gh }}$ does not have to satisfy (114) for arbitrary $\Phi^{\text {gh }}$. It is sufficient for $\mathcal{I}^{\text {gh }}$ to satisfy (114) only for the particular $\Phi^{\text {gh }}$ adopted as a universal ghost factor satisfying (9). In this paper, we simply assume the existence of the state $\mathcal{I}^{\text {gh }}$ satisfying these conditions.

Let us discuss gauge transformations in the form $U=U^{\text {mat }} \otimes \mathcal{I}^{\text {gh }}$. In this case, the transformation (12) is reduced into

$$
\Phi^{\prime \text { mat }}=\left(U^{\text {mat }}\right)^{-1} * \Phi^{\text {mat }} * U^{\text {mat }} .
$$


We will later show that in vacuum string field theory the gauge transformations of Wilson lines are reproduced by the transformation (15) of the sliver state. This is different from Witten's string field theory, in which $U(1)$ gauge transformations are induced by the first term in (12) with $Q$ replaced by the BRS charge $Q_{B}$.

\section{Global gauge transformations of Wilson lines}

From now on, we concentrate only on the matter part of string fields and we drop the symbol 'mat'.

In vacuum string field theory, D-brane is represented by the sliver state $\Xi$. It is represented as a squeezed state in oscillator formalizm. 11, 20

$$
\Xi \equiv \mathcal{N}_{\Xi} e^{-\frac{1}{2} a^{\dagger \mu} C T a^{\dagger \mu}}|0\rangle
$$

The matrix $T$ is given by

$$
T=\frac{1}{2 M}(M+1-\sqrt{(1-M)(1+3 M)}) .
$$

The normalization constant $\mathcal{N}_{\Xi}$ is

$$
\mathcal{N}_{\Xi}=[\operatorname{det}(1-M)(1+T)]^{D / 2},
$$

where $D$ denotes the spacetime dimension 26 .

Now, we suggest the following state as one representing a D-brane with Wilson line.

$$
\left|\Xi_{f}\right\rangle=\mathcal{N}_{\Xi}(f) e^{-f_{m}^{\mu} a_{m}^{\dagger \mu}}|\Xi\rangle
$$

$f_{m}^{\mu}$ is a vector representing the amount of the Wilson line and $\mathcal{N}_{\Xi}(f)$ is a normalization factor depending on $f_{m}^{\mu}$. Due to the reality condition, $f_{m}^{\mu}$ satisfies $f^{\mu *}=-C f^{\mu}$. The fluctuation part of this state reproduces the zero-momentum limit of the HK vector state 13. We can easily show that this is indeed a projector if we take the following normalization.

$$
\mathcal{N}_{\Xi}(f)=\mathcal{N}_{\Xi} \exp \left(\frac{1}{2} f^{\mu} \frac{1}{1-T} C f^{\mu}\right) .
$$

The HK vector state involves a polarization vector with infinite number of components. Similarly, our Wilson line state is parameterized by the vector $f_{m}^{\mu}$. Because of the level index $m$ this vector has infinite number of components. Of cause only one spacetime vector component of $f_{m}^{\mu}$ should be identified with the Wilson line. Others must be unphysical gauge degrees of freedom. In this section, we show that we can gauge away all the degrees of freedom of $f_{m}^{\mu}$ except one spacetime vector component. Let us consider the following gauge transformation specified by a vector $g_{m}$.

$$
U=I_{g}=\mathcal{N}_{I}(g) e^{-\frac{1}{2} a^{\dagger \mu} C a^{\dagger \mu}-g^{\mu} a^{\dagger \mu}}|0\rangle,
$$


where $\mathcal{N}_{I}(g)$ is a normalization factor depending on the vector $g_{m}^{\mu}$. $g_{m}^{\mu}$ is subject to the reality condition $g^{\mu *}=-C g^{\mu}$. By demanding $I_{0}=I_{-g} * I_{g}$ and $\mathcal{N}(g)=$ $\mathcal{N}(-g)$, the normalization factor is uniquely determined as

$$
\mathcal{N}_{I}(g)=\operatorname{det}(1-M)^{D / 2} \exp \left(-\frac{1}{4} g^{\mu} C g^{\mu}\right)
$$

$I_{g}$ normalized in this way satisfies

$$
I_{g_{1}} * I_{g_{2}}=e^{g_{1} \times g_{2}} I_{g_{1}+g_{2}},
$$

where $g_{1} \times g_{2}$ is an antisymmetric product of two vectors $g_{1}$ and $g_{2}$ defined by

$$
g_{1}^{\mu} \times g_{2}^{\mu}=g_{1}^{\mu} C \frac{M_{+}-M_{-}}{2(1-M)} g_{2}^{\mu} .
$$

$I_{0}$ is the matter identity. If we put $g_{1}^{\mu}=t_{1} g^{\mu}$ and $g_{2}^{\mu}=t_{2} g^{\mu}$ with some vector $g^{\mu}$ and real numbers $t_{1}$ and $t_{2}$, (23) reduces to $I_{t_{1} g} * I_{t_{2} g}=I_{\left(t_{1}+t_{2}\right) g}$. This implies that $I_{g}$ is represented as $I_{g}=\exp \Lambda$ where $\Lambda$ is defined by

$$
\Lambda=\left.\frac{d I_{t g}}{d t}\right|_{t=0}=-g^{\mu} a^{\dagger \mu}|I\rangle .
$$

Products of $\Xi_{f}$ and $I_{g}$ are

$$
\Xi_{f} * I_{g}=e^{A(f, g)} \Xi_{f+g(1+T) \rho_{R}}, \quad I_{g} * \Xi_{f}=e^{A(f, g)} \Xi_{f+g(1+T) \rho_{L}},
$$

where

$$
A(f, g)=-f^{\mu} \frac{1}{1-T} C g^{\mu}-\frac{1}{4} g^{\mu} \frac{1+T}{1-T} C g^{\mu} .
$$

The matrices $\rho_{L}$ and $\rho_{R}$ in (27) are the half string projection matrices defined by [21]

$$
\rho_{L}=\frac{M_{-}+T M_{+}}{(1-M)(1+T)}, \quad \rho_{R}=\frac{M_{+}+T M_{-}}{(1-M)(1+T)} .
$$

These satisfy the following equations.

$$
\rho_{L}+\rho_{R}=1, \quad \rho_{L}^{2}=\rho_{L}, \quad \rho_{R}^{2}=\rho_{R}, \quad C \rho_{L} C=\rho_{R} .
$$

Using (26), the gauge transformation of the Wilson line state $\Xi_{f}$ by the deformed identity $I_{g}$ is given as

$$
I_{-g} * \Xi_{f} * I_{g}=\Xi_{f^{\prime}}
$$

where new vector $f_{m}^{\prime \mu}$ after the transformation is

$$
f^{\prime}=f+g(1+T)\left(\rho_{R}-\rho_{L}\right) .
$$

Because this is global transformation without momentum, this does not change the $U(1)$ gauge field on a D-brane. By this global gauge transformation, we can 
gauge away almost degrees of freedom of the vector $f_{m}^{\mu}$. Only components within the kernel of the matrix $(1+T)\left(\rho_{R}-\rho_{L}\right)$ are invariant under the transformation. In fact, the matrix has one dimensional kernel. This can be seen by moving to a basis diagonalizing the matrices $M$ and $M_{ \pm}$. In [22], diagonalized form of the Neumann matrices and an associated complete system of eigenvectors $v_{m}(\kappa)$ labeled by a real number $\kappa$ are given. We can expand the vector $f_{m}^{\mu}$ by this complete basis as

$$
f_{m}^{\mu}=\int f^{\mu}(\kappa) v_{m}(\kappa) d \kappa
$$

Using explicit form of the diagonalized Neumann matrices [22], we can rewrite the transformation law (31) as

$$
f^{\prime \mu}(\kappa)=f^{\mu}(\kappa)+\frac{2 \sinh (\pi \kappa / 2)}{1+e^{\pi|\kappa| / 2}} g^{\mu}(\kappa) .
$$

Therefore, the kernel is generated by $\kappa=0$ eigenvector $v_{m}(0)$ and the Wilson line is identified to $f^{\mu}(\kappa=0)$ up to the normalization. This vector is twist odd. This is consistent with the fact that the vector field on a D-brane is twist odd.

\section{Local gauge transformations of Wilson lines}

We have shown that only one spacetime vector degree of freedom of $f_{m}^{\mu}$ survives from the global gauge transformation (21). In this section, we show that this mode is actually transformed in a way that $U(1)$ gauge field is transformed under local gauge transformations. We consider following string field with non-zero momentum $k^{\mu}$ as a local gauge transformation.

$$
I_{g, k}=e^{i k^{\mu} \widehat{x}^{\mu}} I_{g}
$$

The product of two different string fields of this kind is

$$
I_{f_{1}, p_{1}} * I_{f_{2}, p_{2}}=e^{\left(f_{1}, p_{1}\right) \times\left(f_{2}, p_{2}\right)} I_{f_{1}+f_{2}, p_{1}+p_{2}} .
$$

where the anti-symmetric product involving momenta is defined by

$$
\left(f_{1}^{\mu}, p_{1}^{\mu}\right) \times\left(f_{2}^{\mu}, p_{2}^{\mu}\right)=f_{1}^{\mu} \times f_{2}^{\mu}-v_{1} \frac{1}{1-M}\left(p_{1}^{\mu} f_{2}^{\mu}-p_{2}^{\mu} f_{1}^{\mu}\right) .
$$

We also define non-zero momentum states $\Xi_{f, p}$ by

$$
\Xi_{f, p}=e^{i p^{\mu} \widehat{x}^{\mu}} \Xi_{f}
$$

To compute local gauge transformations generated by $I_{g, k}$, we use the following formulae:

$$
\Xi_{f, p} * I_{g, k}=e^{A(f, p, g, k)} \Xi_{f^{\prime}, p+k}, \quad I_{C g, k} * \Xi_{C f, p}=e^{A(f, p, g, k)} \Xi_{C f^{\prime}, p+k},
$$


where $A(f, p, g, k)$ and $f^{\prime \mu}$ is given by

$A(f, p, g, k)=A(f, g)-\left(p^{\mu}+k^{\mu}\right) v_{1} \frac{1}{1-M} g^{\mu}, \quad f^{\prime \mu}=f^{\mu}-k^{\mu} v_{1} \frac{1-T}{1-M}+g^{\mu}(1+T) \rho_{R}$.

Combining two equations in (38), we easily obtain the transformation law of Wilson line state as $I_{-g,-k} * \Xi_{f} * I_{g, k}=\Xi_{f^{\prime}}$ with the transformed vector $f_{m}^{\prime \mu}$ given by

$$
f^{\prime \mu}=f^{\mu}+g^{\mu}(1+T)\left(\rho_{R}-\rho_{L}\right)-2 k^{\mu} v_{1} \frac{1-T}{1-M} .
$$

The second term on the right hand side in (40) is a contribution of global gauge transformation specified by the parameter $g_{m}^{\mu}$ and this is the same with (31). What is important here is the third term proportional to the momentum $k^{\mu}$. This term is rewritten in the following form in the diagonalized basis:

$$
\delta f^{\mu}(\kappa)=-4 \sqrt{2} k^{\mu} \frac{\sinh (\pi \kappa / 2)}{\kappa(1+\exp (\pi|\kappa| / 2))} .
$$

In contrast to the global transformation (33), this transformation changes the zero-mode $f^{\mu}(\kappa=0)$. Thus, we can identify this transformation to the ordinary gauge transformation of the $U(1)$ gauge field on a D-brane. For example, we can define a vector $A_{\mu}$ by

$$
A_{\mu}=\frac{1}{\sqrt{2} \pi} f_{\mu}(\kappa=0) .
$$

$A_{\mu}$ represents the constant modes of the $U(1)$ gauge field on a $\mathrm{D}$-brane. Then, the gauge transformation $U=I_{g, k}$ reproduces the following gauge transformation of $A_{\mu}$.

$$
A_{\mu}^{\prime}=A_{\mu}-k_{\mu}
$$

Note that this gauge transformation fixes the normalization of the gauge field $A_{\mu}$. The corresponding normalized zero-momentum HK vector state is given by

$$
\Psi=\left.d_{m}^{\mu} \frac{\delta \Xi_{f}}{\delta f_{m}^{\mu}}\right|_{f=0}
$$

with the polarization vector $d_{m}^{\mu}$ satisfying $d^{\mu}(\kappa=0)=\sqrt{2} \pi A^{\mu}$. (We use $\Psi$ to represent the fluctuation part of the string field $\Phi$.)

As a more evidence for our Wilson line state indeed to represent Wilson lines, we can check the shift of the Kaluza-Klein momenta due to Wilson lines. To obtain a non-trivial result, we should extend the gauge group to higher rank one. One way to have higher rank gauge group is to introduce a Chan-Paton factor by hand. Let us introduce $U(N)$ Chan-Paton factor indices for each string field. We suppose the D-brane solution is diagonal

$$
\Xi_{i j}=\Xi_{f_{i}} \delta_{i j}
$$


where the vector $\left(f_{i}\right)_{m}^{\mu}$ is related to the Wilson line $A_{\mu}^{i}$ for $i$-th $U(1)$ of the Cartan subgroup via (42). Let us consider a local gauge transformation in the Cartan subgroup. The string field representing such a gauge transformation is

$$
U_{i j}=\delta_{i j} I_{0, p_{i}}
$$

This transforms the Wilson lines on D-branes as

$$
A_{\mu}^{i} \rightarrow A_{\mu}^{i}-p_{\mu}^{i}
$$

By this transformation, non-diagonal components of the fluctuation part of a string field $\Psi_{i j}$ is transformed as

$$
\Psi_{i j} \rightarrow I_{0,-p_{i}} * \Psi_{i j} * I_{0, p_{j}}
$$

Due to the momentum conservation, momentum of $\Psi_{i j}$ is shifted by $p_{j}-p_{i}$. This represents the momentum shift due to the Wilson lines.

\section{Non-zero momentum modes}

Up to now we have considered only constant modes of the vector field on a Dbrane. In this section, we discuss the gauge field with non-zero momentum. The HK vector state with a momentum $p^{\mu}$ and a polarization vector $d_{\mu}^{m}$ is [13]

$$
\Psi=d_{m}^{\mu} a_{m}^{\dagger \mu} \Xi_{p t, p}
$$

where $t_{m}$ is the following vector defined in [13].

$$
t=3 v(1+3 M)^{-1}(1+T) .
$$

The vector state (49) is a solution of the linearized equation of motion:

$$
\Psi-\Xi * \Psi-\Psi * \Xi=0 \text {. }
$$

In the following we discuss gauge transformation of this mode. We show that we can again gauge away extra degrees of freedom of polarization vector $d_{m}^{\mu}$ and specify components corresponding to the physical $U(1)$ gauge field.

Let us consider infinitesimal gauge transformations with a parameter $\Lambda$ given by

$$
\Lambda=\left.h_{m}^{\mu} \frac{\delta I_{g, k}}{\delta g_{m}^{\mu}}\right|_{g=g_{0}} .
$$

This is non-zero momentum version of the transformation (25). The variation of a fluctuation part $\Psi=\Phi-\Xi$ is given by

$$
\delta \Psi=\Xi * \Lambda-\Lambda * \Xi+\mathcal{O}(\Psi)
$$


We consider only the leading term proportional to the background sliver state $\Xi$ and neglect the small contribution from $\Psi$ on the right hand side in (53). To compute this variation, we define

$$
X_{g, k}=\Xi * I_{g, k}-I_{g, k} * \Xi=e^{A_{2}} \Xi_{f_{2}, k}-e^{A_{1}} \Xi_{f_{1}, k},
$$

where

$$
\begin{gathered}
f_{1}^{\mu}=k v_{1} \frac{1-T}{1-M}+g^{\mu}(1+T) \rho_{L}, \quad f_{2}^{\mu}=-k v_{1} \frac{1-T}{1-M}+g^{\mu}(1+T) \rho_{R}, \\
A_{1}=-\frac{1}{4} g^{\mu} \frac{1+T}{1-T} C g^{\mu}+k^{\mu} v_{1} \frac{1}{1-M} g^{\mu}, \quad A_{2}=-\frac{1}{4} g^{\mu} \frac{1+T}{1-T} C g^{\mu}-k^{\mu} v_{1} \frac{1}{1-M} g^{\mu} .
\end{gathered}
$$

To obtain the last expression in (54) we used formulae (38). The variation $\delta \Psi$ is obtained from this $X_{g, k}$ by

$$
\delta \Psi=\left.h_{m}^{\mu} \frac{\delta X_{g, k}}{\delta g_{m}^{\mu}}\right|_{g=g_{0}} .
$$

General gauge transformations in the form of (52) do not keep the wave function in the form of (49). Therefore, we consider special gauge transformations which keep the form of (49). In order for the two terms in (53) to be added to one term and to give the variation in the form of (49), both $f_{1}^{\mu}$ and $f_{2}^{\mu}$ should coincide with $k^{\mu} t$ when $g^{\mu}$ is set to $g_{0}^{\mu}$. In fact, by only requiring $f_{1}^{\mu}=f_{2}^{\mu}$ we can show that they automatically coincide with $k^{\mu} t$. The condition $f_{1}^{\mu}=f_{2}^{\mu}$ requires the vector $g_{0}^{\mu}$ to satisfy

$$
g_{0}^{\mu}(1+T)\left(\rho_{R}-\rho_{L}\right)=2 k^{\mu} v_{1} \frac{1-T}{1-M} .
$$

Formally, this equation can be solved by multiplying $\left(\rho_{R}-\rho_{L}\right) /(1+T)$ on the both sides and we obtain a solution

$$
g_{0}^{\mu}=2 k^{\mu} v_{1} \frac{1-T}{1-M} \frac{\rho_{R}-\rho_{L}}{1+T} .
$$

This, however, is a dangerous manipulation because $1+T$ has zero eigenvalue. We will return to this problem later and we now adopt the (singular) solution (59) temporally. Substituting this solution into (55), we see that $f_{1}^{\mu}$ and $f_{2}^{\mu}$ actually coincide with $k^{\mu} t$.

$$
f_{1}^{\mu}=f_{2}^{\mu}=\frac{1}{2} g_{0}^{\mu}(1+T)=k^{\mu} v_{1} \frac{1-T}{1-M}\left(\rho_{R}-\rho_{L}\right)=k^{\mu} t .
$$

By substituting (54) and (59) into (57), we obtain

$$
\delta \Psi=\left[k^{\mu} t\left(\rho_{R}-\rho_{L}\right) h^{\mu}+h^{\mu}(1+T)\left(\rho_{R}-\rho_{L}\right) a^{\dagger \mu}\right] \Xi_{k t, k}
$$


Because only the twist odd part of the polarization vector $d_{m}^{\mu}$ can include the $U(1)$ vector modes, let us assume the parameter $h_{m}^{\mu}$ of the gauge transformation to be twist even. Then a transformation low of the polarization vector $d_{m}^{\mu}$, which is identical to that for constant modes, is obtained.

$$
\delta d^{\mu}=h^{\mu}(1+T)\left(\rho_{R}-\rho_{L}\right) .
$$

Therefore, just as the constant modes, we claim that the physical vector modes are the $\kappa=0$ components of the polarization vector $d_{m}^{\mu}$.

Now, let us return to the problem about the dangerous manipulation used in obtaining $g_{0}^{\mu}$ in (59). One way to regularize this singularity is to use regularized $g_{0}^{\mu}$. We denote the regularized version of $g_{0}^{\mu}$ as $g_{0 \text { reg. }}^{\mu}$. We take $g_{0 \text { reg }}^{\mu}$ such that its $\kappa$ expansion $g_{0 \text { reg }}^{\mu}(\kappa)$ does not diverge at $\kappa=0$. This regularization influents the gauge transformation of $\Psi$. Especially, the two vectors $f_{1}^{\mu}$ and $f_{2}^{\mu}$ do not coincide with each other. The gauge transformation obtained from $g_{0 \text { reg }}^{\mu}$ is

$$
\delta \Psi=h^{\mu}(1+T)\left[\rho_{R} a^{\dagger \mu} e^{A_{2}} \Xi_{f_{2}, k}-\rho_{L} a^{\dagger \mu} e^{A_{1}} \Xi_{f_{1}, k}\right],
$$

where $f_{1}^{\mu}, f_{2}^{\mu}, A_{1}$ and $A_{2}$ are those in (55) and (56) with $g^{\mu}$ replaced by $g_{0 \text { reg. }}^{\mu}$. (To make the computation simpler, we assumed the orthogonality $h^{\mu} k^{\mu}=0$ and the parallelness $g_{0 \text { reg }}^{\mu} \| k^{\mu}$.) This variation cannot be regarded as a gauge transformation of polarization vector $d_{m}^{\mu}$ in (49). Instead, it can be regarded as a variation of a polarization vector of a 'regularized' wave function $\Psi_{\text {reg }}$.

$$
\Psi_{\text {reg }}=d^{\mu} \rho_{R} a^{\dagger \mu} \Xi_{f_{2}, k}+d^{\mu} \rho_{L} a^{\dagger \mu} \Xi_{f_{1}, k} .
$$

Two vectors $f_{1}^{\mu}$ and $f_{2}^{\mu}$ in (64) are those in (55) defined with $g_{0 \text { reg }}^{\mu}$, and this wave function coincides with the original HK vector state (49) after taking the limit $g_{\text {Oreg }}^{\mu} \rightarrow g_{0}^{\mu}$. For this regularized HK vector state, the gauge transformation (63) induces the transformation (62) for the polarization vector $d_{m}^{\mu}$ of the reguralized state. (To obtain this simple expression, we assumed that $g_{0 \text { reg }}^{\mu}$ and $h^{\mu}$ are twist even and $d^{\mu}$ is twist odd. Then $A_{1}$ is equal to $A_{2}$ and we can drop the factors $e^{A_{1}}$ and $e^{A_{2}}$ by rescaling the gauge transformation parameter $h^{\mu}$.) Therefore, we can again claim that only $\kappa=0$ modes of the polarization vector $d_{m}^{\mu}$ is physical degrees of freedom.

\section{Discussions}

Based on the factorization ansatz of gauge transformations, we have shown that only the $\kappa=0$ components of the polarization vector $d_{m}^{\mu}$ are physical degrees of freedom and all the other components are gauge degrees of freedom. This implies that if we substitute the wave function (49) into the string field action we must obtain an action depending only on the $\kappa=0$ components of the polarization 
vector $d_{m}^{\mu}$. When the gauge field is normalized such that the gauge transformation does not depend on the coupling constant as (43), the coupling constant appears as an overall factor of the action of the gauge field. Therefore, if we obtain the kinetic term of the vector field we can determine the gauge coupling constant as a coefficient of the kinetic term. Unfortunately, however, we cannot obtain the gauge invariant action nor the gauge coupling constant because of a difficulty we will mention below.

Instead of computing the action of the vector field, let us consider the linearized equation of motion because the quadratic part of the action is represented as a BPZ inner product of $\Psi$ and the left hand side of the equation of motion (51). We can easily show that the equation of motion is gauge invariant:

$$
\begin{aligned}
& \delta(\Psi-\Xi * \Psi-\Psi * \Xi) \\
= & \Xi * \Lambda-\Lambda * \Xi-\Xi *(\Xi * \Lambda-\Lambda * \Xi)-(\Xi * \Lambda-\Lambda * \Xi) * \Xi \\
= & \Xi * \Lambda-\Lambda * \Xi-(\Xi * \Xi) * \Lambda+(\Xi * \Lambda) * \Xi-(\Xi * \Lambda) * \Xi+\Lambda *(\Xi * \Xi) \\
= & 0 .
\end{aligned}
$$

We used associativity of $*$-product between the second expression and the third one in (65). On the other hand, if we substitute the solution (49) into the equation of motion, we obtain [13, 14, 15]

$$
\Psi-\Xi * \Psi-\Psi * \Xi=\left(1-e^{G p^{2} / 2}\right) \Psi, \quad G=\log 2 .
$$

This is evidently not gauge invariant and depend on all the components of $d_{m}^{\mu}$. This implies that the above 'proof' of the gauge invariance in (65) is not correct.

In the last section we mentioned that we should regularize the wave function when we consider the gauge transformation of non-zero momentum modes. Namely, more reliable way to compute the gauge invariant equation of motion and the action is to use the regularized wave function (64) and to take the limit $g_{0 \text { reg }}^{\mu} \rightarrow g_{0}^{\mu}$ after all the computation. However, this regularization does not change the situation. The problem is more serious.

To obtain the gauge transformation $\delta \Psi$, we need to compute $*$-product of the sliver $\Xi$ and the deformed identity state $I_{g, k} . \delta \Psi$ is obtained as a derivative of this product. Furthermore, to obtain the variation of the equation of motion, we need to compute $\Xi * \Xi * I_{g, k}$ and similar products in different order. We need the associativity in these products to show the gauge invariance of the equation of motion. However, we can easily show that the associativity is broken in this kind of product.

$$
(\Xi * \Xi) * I_{g, k} \neq \Xi *\left(\Xi * I_{g, k}\right)
$$

This is the reason why we failed to obtain a gauge invariant equation of motion. To obtain the coupling constant correctly as a coefficient of the gauge invariant action, we should resolve this problem. At this point, however, we do not have any reliable way to handle associativity anomaly. 
Finally, let discuss other questions which may be interesting. In the last section, we introduce Chan-Paton factor by hand to discuss the momentum shift due to the Wilson lines. More interesting way to construct multiple D-brane is to use higher rank projector. In [21], construction of higher rank projector as a sum of different rank one projectors is discussed. They use "*-rotation" to make projectors orthogonal to each other. Similarly, our Wilson line solution may be used to make higher rank projector. The BPZ inner product and *-product of two different Wilson line states is

$$
\left\langle\Xi_{f_{1}} \mid \Xi_{f_{2}}\right\rangle=e^{D\left(f_{1}-f_{2}\right)}\langle\Xi \mid \Xi\rangle, \quad \Xi_{f_{1}} * \Xi_{f_{2}}=e^{D\left(f_{1}-f_{2}\right)} \Xi_{f \rho_{L}+g \rho_{R}},
$$

where $D(f)$ is the following function.

$$
D(f)=\frac{1}{2} f^{\mu} \frac{1}{1-T^{2}} C f^{\mu} .
$$

$1-T^{2}$ has one zero eigenvalue at $\kappa=0$ and all the other eigenvalues are positive. We can regularize $D(f)$ by replacing $1-T^{2}$ by $1-T^{2}+\epsilon$ with a positive number $\epsilon$. In the limit $\epsilon \rightarrow+0$, the exponent $D(f)$ of coefficient of right hand side of (68) diverges to minus infinity. Therefore, if two D-brane have different Wilson lines, these state are orthogonal to each other. This implies that $\Xi_{f}+\Xi_{g}$ is a projection operator representing two D-brane solution.

It may be also interesting to study T-duality between Wilson lines on Dbranes and positions of lump solutions [3]. The explicit form of the lump solution is known and we can construct a solution of a lump at an arbitrary position by using the shift operator $e^{i a \widehat{p}}$. One may think that the Wilson line solution studied here would be obtained from the lump solution by T-duality. However, the T-duality between them is highly non-trivial. The gauge fields on D-branes and scalar fluctuation modes of D-branes have opposite sign of twist. Thus, Tduality may be represented as an isomorphy of the $*$-algebra exchanging twist even modes $\left\{a_{2 n}\right\}$ and twist odd modes $\left\{a_{2 n+1}\right\}$.

\section{Acknowledgements}

We would like to thank H. Hata, I. Kishimoto, S. Moriyama and K. Ohmori for valuable discussions.

\section{References}

[1] E. Witten, "Noncommutative Geometry and String Field Theory", Nucl. Phys. B268, 253.

[2] L. Rastelli, A. Sen, B. Zwiebach, "String Field Theory Around the Tachyon Vacuum", hep-th/0012251. 
[3] L. Rastelli, A. Sen, B. Zwiebach, "Classical solutions in string field theory around the tachyon vacuum", hep-th/0102112.

[4] L. Rastelli, A. Sen and B. Zwiebach, "Boundary CFT construction of D-branes in vacuum string field theory", JHEP 0111 (2001) 045, hep-th/0105168.

[5] L. Rastelli, A. Sen, B. Zwiebach, "Vacuum String Field Theory", hep-th/0106010.

[6] A. Sen, B. Zwiebach, "Tachyon condensation in string field theory", JHEP 0003 (2000) 002, hep-th/9912249.

[7] N. Moeller, W. Taylor, "Level truncation and the tachyon in open bosonic string field theory", Nucl.Phys. B583 (2000) 105, hep-th/0002237.

[8] A. A. Gerasimov, S. L. Shatashvili, "On Exact Tachyon Potential in Open String Field Theory”, JHEP 0010 (2000) 034, hep-th/0009103.

[9] W. Taylor, "Mass generation from tachyon condensation for vector fields on D-branes", JHEP 0008 (2000) 038, hep-th/0008033.

[10] I. Ellwood, W. Taylor, "Open string field theory without open strings", Phys.Lett. B512 (2001) 181, hep-th/0103085.

[11] A. Kostelecky and R. Potting, "Analytical construction of a nonperturbative vacuum for the open bosonic string", Phys.Rev. D63 (2001) 046007, hep-th/0008252.

[12] K. Okuyama, "Ratio of tensions from vacuum string field theory", hep-th/0201136.

[13] H. Hata, T. Kawano, "Open String States around a Classical Solution in Vacuum String Field Theory", JHEP 0111 (2001) 038, hep-th/0108150.

[14] H. Hata and S. Moriyama, "Observables as twist anomaly in vacuum string field theory", JHEP 0201 (2002) 042, hep-th/0111034.

[15] L. Rastelli, A. Sen and B. Zwiebach, "A note on a proposal for the tachyon state in vacuum string field theory," JHEP 0202 (2002) 034, hep-th/0111153.

[16] D. J. Gross, A. Jevicki, "Operator formulation of interacting string field theory", Nucl.Phys.B283,1(1987).

[17] D. J. Gross, A. Jevicki, "Operator formulation of interacting string field theory. 2", Nucl.Phys.B287,225(1987). 
[18] I. Kishimoto, "Some properties of string field algebra", JHEP 0112 (2001) 007, hep-th/0110124.

[19] I. Kishimoto, K. Ohmori, "CFT Description of Identity String Field: Toward Derivation of the VSFT Action", hep-th/0112169.

[20] L. Rastelli and B. Zwiebach, "Tachyon potentials, star products and universality", JHEP 0109 (2001) 038, hep-th/0006240.

[21] L. Rastelli, A. Sen and B. Zwiebach, "Half-strings, projectors, and multiple D-branes in vacuum string field theory," JHEP 0111 (2001) 035, hep-th/0105058.

[22] L. Rastelli, A. Sen and B. Zwiebach, "Star algebra spectroscopy", hep-th/0111281.

[23] Y. Okawa, "Open string states and D-brane tension from vacuum string field theory", th/0204012. 